\title{
Accurate Direction-of-Arrival Estimation Method based on Space-Time Modulated Metasurface
}

This paper was downloaded from TechRxiv (https://www.techrxiv.org).

\section{LICENSE}

CC BY 4.0

SUBMISSION DATE / POSTED DATE

$28-12-2021 / 05-01-2022$

\section{CITATION}

Li, Mengmeng (2022): Accurate Direction-of-Arrival Estimation Method based on Space-Time Modulated Metasurface. TechRxiv. Preprint. https://doi.org/10.36227/techrxiv.17698181.v1

$\mathrm{DOI}$

10.36227/techrxiv.17698181.v1 


\title{
Accurate Direction-of-Arrival Estimation Method based on Space-Time Modulated Metasurface
}

\author{
Xinyu Fang, Mengmeng Li, Senior Member, IEEE, Juzheng Han, \\ Davide Ramaccia, Senior Member, IEEE, Alessandro Toscano, Senior Member, IEEE, \\ Filiberto Bilotti, Fellow, IEEE, and Dazhi Ding, Senior Member, IEEE
}

\begin{abstract}
In this paper, we present a metasurface-based Direction of Arrival (DoA) estimation method that exploits the properties of space-time modulated reflecting metasurfaces to estimate in real-time the impinging angle of an illuminating monochromatic plane wave. The approach makes use of the amplitude unbalance of the received fields at broadside at the frequencies of the two first-order harmonics generated by the interaction between the incident plane wave and the modulated metasurface. Here, we first describe analytically how to generate the desired higher-order harmonics in the reflected spectrum and how to realize the breaking of the spatial symmetry of each order harmonic scattering pattern. Then, the one dimensional (1D) omnidirectional incident angle can be analytically computed using +1 st and -1st order harmonics. The approach is also extended to 2D DoA estimation by using two orthogonally arranged 1D DoA modulation arrays. The accuracy of 1D DoA estimation is verified through full-wave numerical simulations. Compared to conventional DoA estimation methods, the proposed approach simplifies the computation and hardware complexity, ensuring at the same time estimation accuracy. The proposed method may have potential applications in wireless communications, target recognition, and identification.
\end{abstract}

Index Terms - DoA estimation, Space-time-modulation, metasurface.

\section{INTRODUCTION}

$\mathrm{D}$ IRECTION of Arrival (DoA) estimation is a key functionality in radar tracking and radio navigation [1][2], satellite communications on-the-move (SOTM) [3], and smart antenna [4]-[6] systems. It is fundamental for establishing and maintaining communication between two terminals while one

Manuscript received xxxx. This work was supported in part by Natural Science Foundation of China of 61871222, 62025109, and 61890541, the Fundamental Research Funds for the Central Universities of 30921011101. FB acknowledges the support of the research contract MANTLES funded by the Italian Ministry of University and Research, PRIN 2017 n. 2017BHFZKH.

X. Y. Fang, M. Li, J. Z. Han, and D. Z. Ding are with the Department of Communication Engineering, Nanjing University of Science and Technology, Nanjing, China (e-mail: limengmeng@njust.edu.cn; hanjuzheng@njust.edu.cm dzding@njust.edu.cn).

D. Ramaccia, A. Toscano, and F. Bilotti are with the Department of Industrial, Electronic and Mechanical engineering, ROMA TRE University, 00146 Rome, Italy (e-mail: davide.ramaccia@uniroma3.it; alessandro.toscano@uniroma3.it; filiberto.bilotti@uniroma3.it). is moving with respect to the other. Moreover, it is also needed for the next generations of mobile communication, such as $5 \mathrm{G}$ and beyond $5 \mathrm{G}(\mathrm{B} 5 \mathrm{G})$, and the future sixth-generation $(6 \mathrm{G})$ communication, enabling location services for the mobile Internet [7][8]. In this framework, array signal processing methods are the most common techniques to estimate DoA. Among them, it is worth mentioning techniques based on minimum variance distortion-less response (MVDR) [9], estimation of signal parameters via a rotational invariant (ESPRIT) [10][11], multiple signal classification (MUSIC) [12][13], and Bayesian compressive sensing (BCS) [14]. The high performances reached by these techniques are, however, counterbalanced by the need of massive arrays and the use of multiple sensors or channels, intense calculation, and expensive hardware. In this framework, a low in transmission and reflection, such as amplitude [15], polarization [16], propagation direction [17][18], and, more recently, the frequency content [19]-[22] -cost technology and low-complexity DoA estimation system would represent an important advance in those applications where the angular localization of the transmitter, interfering signals, and users is mandatory.

In the last decades, metasurfaces have demonstrated to be a breakthrough technology in a number of applications, spanning from microwave to optical frequency ranges [23]-[25]. They typically consist of a bi-dimensional (2D) periodic array of subwavelength meta-atoms able to control the properties of the incident electromagnetic field. In particular, the control of the spatial and/or temporal characteristics of the interacting fields was made possible thanks to the development of dynamically reconfigurable metasurfaces, using different reconfiguring technologies based on pin diodes [26][27], varactors [28]-[30], MEMS [31], graphene [32], and liquid crystal [33]. The control can be performed through analogical control signals [20]-[22] or via digital control [34]-[36]. In both cases, the resulting metasurfaces belong to the wider family of space-time metamaterials [37]-[42], which exhibit unprecedented capabilities and find applications in different operative scenarios, such as non-reciprocal structures [19], [43]-[46], arbitrary multi-bit programmable systems [47], wireless communications [48]-[50], and spread-spectrum radar camouflaging [51]. 


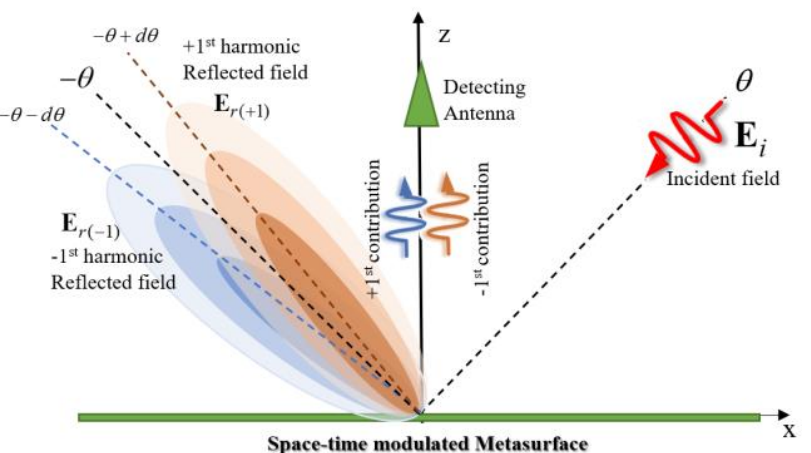

Figure 1. Illustration of the operative scenario of a space-time modulated metasurface for DoA estimation.

In this paper, we present and discuss a metasurface-based DoA method that exploits the properties of space-time modulated reflecting metasurfaces to estimate in real-time the impinging angle of an illuminating monochromatic plane wave at frequency $\omega_{0}$. The system is composed by a space-time modulated metasurface and a detecting antenna placed in the far-field region just above it. The DoA estimation is here performed by measuring the amplitude unbalance of the reflected fields at the frequencies of the two first-order harmonics, i.e., $\omega_{0} \pm \omega_{p}$, generated by the interaction between the incident plane wave and the metasurface modulated at frequency $f_{p}=\omega_{p} / 2 \pi$. However, this approach, though being promising as such, it is practically useless, due to the typical frequency used for the modulation, i.e. $\omega_{p} \square \omega_{0}$, which makes the two harmonics exhibiting an almost identical amplitude, being governed by the Manley-Rowe relations [52][53]. Here, we exploit the spatial modulation as an additional degree-of-freedom for enhancing the required unbalancing, inducing a pattern asymmetry for the fields scattered at the two first-order harmonics.

The proposed approach exhibits significant advantages with respect to the mentioned common array signal processing methods (MVDR, ESPRIT, MUSIC, BCS). Indeed, these systems require massive array antennas for achieving good performances. More recently, time modulated arrays with a limited number of antenna elements have been also proposed for DoA estimation [54][55] with the goal to reduce the number of antennas and overall computational complexity. However, the hardware of the backend network is still complex, involving properly connected phase shifters, switches, and power combiners. On the contrary, the metasurface-based DoA system shifts most part of the computational efforts at the electromagnetic level, reducing, thereby, the latency in detection and removing the need of a complex circuity. Both the frequency modulation and the spatial unbalancing needed for estimating the DoA is here performed mainly at the metasurface level, whereas the remaining computation is extremely low-cost and consists only in the evaluation of the relative difference in amplitude of the two first-order harmonics received by the fixed detecting antenna. In the next sections, we describe the analytical model used for the DoA estimation and demonstrate through a proper set of numerical simulations the high accuracy reached by this method.

The paper is organized as follows. After having illustrated the basic idea, in Sec. II, we derive analytically the temporal modulation scheme to apply to a space-time modulated metasurface for generating the two desired first-order harmonics and the spatial modulation scheme for achieving the breaking of the spatial symmetry of each order harmonic scattering pattern. In Sec. III, the analytical DoA estimation method based on the double-sideband (DSB) space-time-modulated metasurface is derived for both 1D and 2D case. In Sec. IV, a realistic metasurface is used for demonstrating numerically the accuracy of the proposed method for DoA estimation. Different configurations have been tested, showing the operative bounds of the proposed system. Finally, in Sec. V, a short conclusion of our work is given.

\section{SPACE-Time modulated Metasurface FOR DOA ESTIMATION}

In this Section, we present the proposed DoA estimation method based on space-time coding modulated metasurfaces. After having introduced the basic idea, we analytically describe the realization of the DSB time modulation by using a 1-bit coded metasurface, which allows exciting the required $\pm 1^{s t}$ harmonics in reflection. The breaking of the spatial symmetry of each order harmonic scattering pattern is achieved by applying a delayed copy of the modulating signal to adjacent portions of the space-time modulated metasurface.

\section{A. Basic idea of DoA estimation}

Let us consider the operative scenario reported in Fig. 1. A space-time modulated metasurface is illuminated by a monochromatic plane wave at frequency $\omega_{0}$ propagating with an angle $\theta$ with respect to the positive $z$-direction.

We assume that the metasurface-based DoA system operates only on the $x z$-plane. This constrain will be relaxed later. Regardless the technology used for imparting the space-time modulation on the metasurface, when its surface properties are temporally modulated with frequency $\omega_{p} \square \omega_{0}$, a frequency mixing between the incident and modulating signals takes place, spreading the energy of the incident field over a number of frequency harmonics located at $\omega_{0} \pm k \omega_{p}$, with $k=1,2,3, \ldots$. In case of temporally modulated spatial uniform metasurfaces [20][21], the reflected field propagates towards the specular-direction $(-\theta)$. However, the metasurfaces can be also modulated in space, allowing the frequency contributions of the reflected field being radiated towards different directions. This allows obtaining a spatial splitting of the radiation patterns, as demonstrated by several works on space-time modulated metastructures [34][36][42][48]. In Fig. 1, the radiation patterns of the two first-harmonics at $\omega_{0} \pm \omega_{p}$ are shown, one pointing towards $-\theta+d \theta$ and one towards $-\theta-d \theta$. The detecting antennas placed in the far-field region just above the metasurface can now receive a different amplitude contribution at the two frequencies $\omega_{0} \pm \omega_{p}$ and estimate the DoA of the 
incident plane wave based on the relative unbalancing of such values.

\section{B. DSB modulation in time coding metasurfaces}

The main purpose of this part is to show analytically how a time-varying reflecting metasurfaces can generate the required harmonics in the reflected spectrum for applying the proposed method for DoA estimation. Since we are here interested in the temporal modulation of the incident wave, we can relax the constrain on the oblique illumination condition and assume that the metasurface is normally illuminated by the plane wave. In the next sections, we will consider also the effect of the oblique incidence.

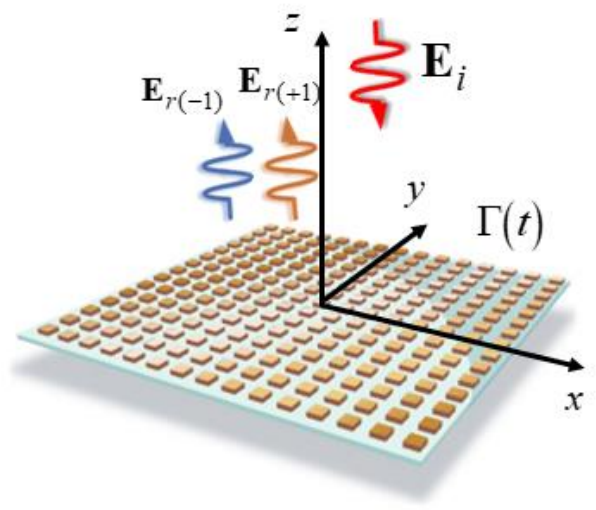

Figure 2. Double-side band modulating metasurface illuminated by a normally incident plane wave able to reflect a plane wave consisting of a superposition of the two first-harmonics.

In Fig. 2, we report an ideal non-penetrable (i.e. characterized by a zero-transmission) reflecting metasurface, whose macroscopic response can be modeled through its time-varying reflection coefficient $\Gamma(t)$. The metasurface is illuminated by a normally incident plane wave of the form:

$$
\mathbf{E}_{i}(z, t)=\mathbf{E}_{0} e^{j k_{0} z} e^{j \omega_{0} t},
$$

and the desired reflected field consists of the superposition of the two first-order harmonics at frequencies $\omega_{0} \pm \omega_{p}$ with the same amplitude:

$$
\mathbf{E}_{r}(z, t)=\mathbf{E}_{r} e^{-j k_{0} z}\left[e^{j\left(\omega_{0}+\omega_{p}\right) t}+e^{j\left(\omega_{0}-\omega_{p}\right) t}\right],
$$

At the metasurface location, i.e. $z=0$, the incident and reflected fields are related as follows:

$$
\mathbf{E}_{r}(0, t)=\mathbf{E}_{i}(0, t) \cdot \Gamma(t),
$$

where $\Gamma(t)$ is the degree-of-freedom we use to achieve the generation of two equal amplitude harmonics at $\omega_{0} \pm \omega_{p}$.

Combining Eqs. (1)-(3), the reflection coefficient $\Gamma(t)$ can be expanded in the form:

$$
\bar{u}(t)=\frac{E_{r}}{E_{0}}\left(e^{j \omega_{p} t}+e^{-j \omega_{p} t}\right)=2 \frac{E_{r}}{E_{0}} \cos N_{p} t
$$

To satisfy the passivity constrain, in Eq. (4), the amplitude of the reflected field of each of the two first-order harmonics is half compared to the one of the incident field, i.e., $E_{r}=E_{0} / 2$. This implies that half of the overall impinging energy is distributed between the two first order harmonics at $\omega_{0} \pm \omega_{p}$, whereas the rest is necessary dissipated by the intrinsic reflective response of the metasurface with cosine temporal profile due to the zeros of the cosine function. Despite the cosine temporal profile of the reflection coefficient ensures theoretically the required response from the metasurface, such a temporal profile needs a continuous modulation of the surface properties of the metasurface, as demonstrated in [20] [21], which is not easy to achieve.

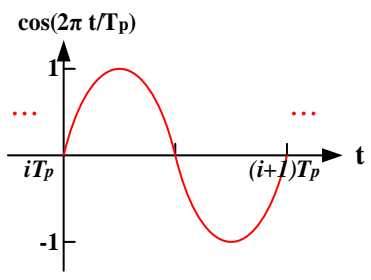

(a)

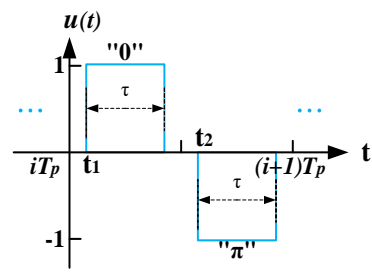

(b)
Figure 3. (a) Temporal signal with cosine profile to be used for achieving a perfect double-side band excitation at frequencies $\omega_{0} \pm \omega_{p}$, (b) approximate three state signal used to match the curves in (a).

To simplify the metasurface implementation, the cosine temporal profile of the reflection coefficient can be approximately replaced with acceptable tolerance by a train of rectangular pulses [56], as shown in Fig. 3. The time-period $T_{p}$ is the modulation period corresponding to the angular frequency $\omega_{p}=2 \pi / T_{p}$. The rectangular pulses of amplitudes " +1 " and " -1 " in Fig. 3(b) correspond to the reflection phases " 0 " or " $\pi$ ", respectively. Now, the start time $t_{1}$ of the " +1 " state, the start time $t_{2}$ of the " -1 " state, and the pulse duration $\tau$ characterizing the shape of the rectangular pulses must be analytically derived to match the cosine time profile as better as possible [56], and ensure the excitation of only the two first-order harmonics.

For the first time-period $T_{p}$ starting at $t=0$, the rectangular time sequence can be expressed as:

$$
u(t)=\left\{\begin{array}{cr}
+1, & t_{1} \leq t \leq t_{1}+\tau \\
-1, & t_{2} \leq t \leq t_{2}+\tau \\
0, & \text { others within }\left[0 ; T_{p}\right]
\end{array},\right.
$$

The periodic time sequence is decomposed by using the Fourier series to highlight the contribution of the different frequency harmonics as:

$$
u(t)=\sum_{h=-\infty}^{+\infty} a_{h} e^{j h \omega_{p} t}, \text { with } h=0, \pm 1, \ldots \pm \infty
$$

where $h$ is the order of the specific harmonic, and $a_{h}$ is the corresponding Fourier coefficients, whose expression is evaluated as follows:

$$
a_{h}=\frac{1}{T_{p}} \int_{0}^{T_{p}} u(t) e^{-j h \omega_{p} t} d t .
$$

Considering the periodicity of the modulation, we can normalize the temporal axis with respect to the time-period $T_{p}$ and obtain the dimensionless quantities: 


$$
\tilde{t}_{1}=t_{1} / T_{p}, \quad \tilde{t}_{2}=t_{2} / T_{p}, \quad \tilde{\tau}_{1}=\tau / T_{p} .
$$

Eq. (7) can now be easily evaluated as follows:

$$
\begin{array}{rlr}
a_{h} & =\int_{0}^{1} u(t) e^{-j 2 \pi h \tilde{t}} d \tilde{t}= \\
& =\left(\int_{\tilde{t}_{1}}^{\tilde{t}_{1}+\tilde{\tau}} e^{-j 2 \pi h \tilde{t}} d \tilde{t}-\int_{\tilde{t}_{2}}^{\tilde{t}_{2}+\tilde{\tau}} e^{-j 2 \pi h \tilde{t}} d \tilde{t}\right) \\
& = \begin{cases}0, & h=0 \\
\frac{2 j}{h \pi} \sin (h \pi \tilde{\tau}) \sin \left[h \pi\left(\tilde{t}_{2}-\tilde{t}_{1}\right)\right] e^{-j h \pi\left(\tilde{t}_{2}+\tilde{t}_{1}+\tilde{\tau}\right)}, & h \neq 0\end{cases}
\end{array}
$$

Considering the term $\sin \left[h \pi\left(\tilde{t}_{2}-\tilde{t}_{1}\right)\right]$, when $\tilde{t}_{2}-\tilde{t}_{1}=0.5$, all the even order harmonics vanish, i.e., $\left|a_{h}\right|=0$ for $h=2 k, k \in \square$. Moreover, considering the term: $\sin (h \pi \tilde{\tau})$, setting $\tilde{\tau}=1 / 3$, the $h^{\text {th }}$-order harmonics with $h=3 \mathrm{k}, \mathrm{k} \in \mathbb{Z}$ also vanish. In this case, only few harmonics far from the $\pm 1^{\text {st }}$ ones survive, e.g. the $\pm 5^{\text {th }}, \pm 7^{\text {th }}, \pm 11^{\text {th }}$, and so on. This configuration ensures the highest isolation between the first order harmonic and the other order harmonics achievable with a binary time modulated metasurface. However, it is worth noticing that, to exactly match the temporal profile shown in in Fig. 3(b), an absorption state is required between two consecutive opposite pulses.

To further simplify the implementation, we remove the possibility to have an absorption state, setting $\tilde{\tau}=0.5$. This leads to a time-varying reflection coefficient that can exhibit only " +1 " and " -1 " reflection states, which corresponds to the reflection phases " 0 " and " $\pi$ ", respectively, as shown in Fig. 4(a), over a time-period $T_{p}$. Consequently, the presence of the $h^{\text {th }}$-order harmonics with $h=3 \mathrm{k}, \mathrm{k} \in \mathbb{Z}$, is restored. Imposing $\tilde{t}_{2}-\tilde{t}_{1}=0.5$ and $\tilde{\tau}=0.5$ into Eq. (9), the complete set of the $a_{h}$ coefficients is:

$$
a_{h}=\frac{2 j}{h \pi} \sin ^{2}\left(\frac{h \pi}{2}\right) e^{-j h \pi\left(2 \tilde{t}_{1}+1\right)} \cdot \operatorname{sgn}(h),
$$

where $\operatorname{sgn}(h)$ is the sign function. In Fig. 4(b), we report the amplitude of the harmonic coefficients as a function of the harmonic order. It can be found that the amplitudes of the first-order harmonics are still three times bigger than the other order harmonics, letting our approach to still benefit of the presence of strong $\pm 1^{s t}$-order harmonics.

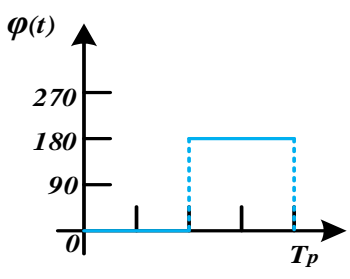

(a)

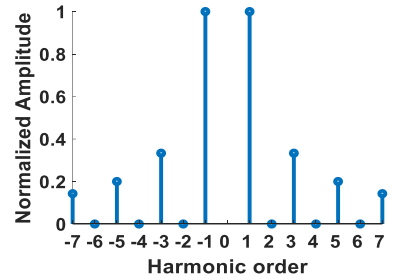

(b)
Figure 4. (a) The time-varying phase profile $\varphi(t)$ of the time sequence $u(t)$ to achieve DSB modulation. (b) The normalized coefficient amplitude of the generated harmonics.

In the next Section, the required spatial modulation is imposed on the metasurface in order to achieve the splitting of the radiation patterns of the reflected $1^{\text {st }}$-order harmonics, which allows estimating the DoA of the incident wave.

\section{Pattern unbalancing in DSB modulated metasurface}

Once the proper temporal modulation of the 1-bit space-time metasurface is properly defined (see Section II-B for further details), the spatial asymmetry must be introduced for splitting the radiation patterns of the $\pm 1^{s t}$-order harmonics and enable the DoA estimation capability of the system. The metasurface is partitioned in areas of width $D$ in $x$-direction, called sub-macrocells, each of which covers the entire extension along $y$-direction, including $N_{x} \times N_{y}$ lines of space-time modulated inclusions, as shown in Fig. 5(a).

The spatial asymmetry is here introduced by applying different modulation signals to adjacent sub-macrocells, i.e., "Sub-macrocell 1" modulated by the signal $u_{1}(t)$, and "Sub-macrocell 2", modulated by the signal $u_{2}(t)$, reported in Fig. 5(b) and 5(c), respectively. The two signals are identical, except for a minor time delay $\Delta t$. They compose the DoA-MTS macro unit-cell responsible for the space-time asymmetry in the reflected patterns. It is worth mentioning that, due to the uniformity along $y$-direction, the scheme in Fig. 5(a) can perform DoA estimation only for plane waves propagating on the $x z$-plane.

Since the proposed method is valid regardless the specific technology and subwavelength inclusion composing the metasurface, in the following we describe its scattering pattern in terms of the Array Factor. The complete scattering response can be easily obtained by multiplying the array factor we derive in the following and the radiation pattern of the single subwavelength inclusion composing the metasurface [57]. The array factor of the single DoA-MTS macro unit-cell for the $h$-th harmonic is indicated as $a f_{h}(\theta, \varphi)$ and can be written as the product of the array factors of the arrays of subwavelength metasurface unit-cell in the $x$ - and $y$-directions:

$$
\begin{aligned}
a f_{h}(\theta, \phi) & =\sum_{i=1}^{2} \sum_{n_{x}=1}^{N_{x}} a_{h}^{(i)} e^{j k_{0}\left[\left(n_{x}-1\right) p_{x}+(i-1) D\right] \sin \left(\theta+\theta_{i n c}\right) \cos \left(\phi+\phi_{i n c}\right)} \times \\
& \times \sum_{n_{y}=1}^{N_{y}} e^{j k_{0}\left(n_{y}-1\right) p_{y} \sin \left(\theta+\theta_{i n c}\right) \sin \left(\phi+\phi_{i n c}\right)} .
\end{aligned}
$$

In (11), the parameters $p_{x, y}$ are the periodicities of the subwavelength inclusions in $x$ - and $y$-direction, respectively, $k_{0}$ is the free-space wavevector of the incident wave, $\theta_{i n c}, \phi_{i n c}$ identify the oblique incident angle of the illuminating wave.

The weighting coefficient $a_{h}^{(i)}$ is the complex amplitude of the $h$-th harmonic generated by the modulated subwavelength modulated inclusions within the Sub-macrocell $i$. With reference to Fig. 3(b) and Figs. 5(b)-(c), for identifying the starting time of the modulating signals of the two Sub-macrocells, the corresponding complex amplitudes are:

$$
a_{h}^{(i)}= \begin{cases}\frac{2 j}{h \pi} \sin ^{2}\left(\frac{h \pi}{2}\right) e^{-j h \pi\left(2 \tilde{t}_{1}^{(1)}+1\right)} \cdot \operatorname{sgn}(h), & i=1 \\ \frac{2 j}{h \pi} \sin ^{2}\left(\frac{h \pi}{2}\right) e^{-j h \pi\left(2 \tilde{t}_{1}^{(2)}+1\right)} \cdot \operatorname{sgn}(h), & i=2\end{cases}
$$



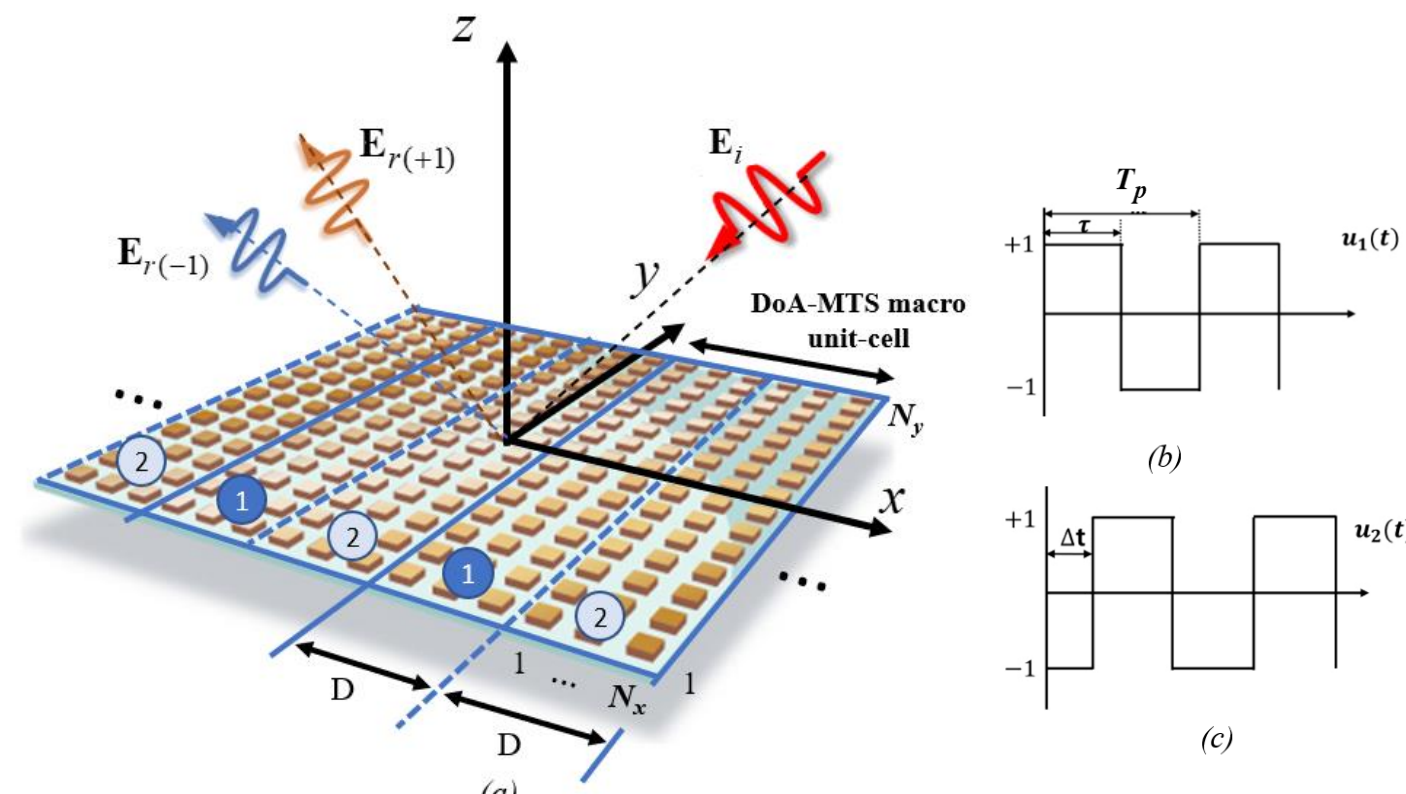

(b)

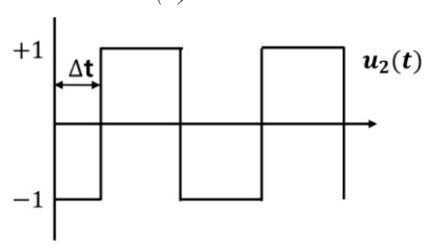

(c)

(a)

Figure 5. (a) Sketch of the space-time modulation scheme to apply to a double-side band modulating metasurface illuminated by an incident plane wave able to reflect a plane wave consisting of a superposition of the two first-order harmonics. (b)-(c) Temporal signals used for modulating the two sub-macrocells composing the DoA-MTS macrounit cell.

In (12), when $i=1$, the complex valued coefficient $a_{h}$ is obtained for $\tilde{t}_{1}^{(1)}=\tilde{t}_{1}$, whereas, when $i=2$, the complex valued coefficient $a_{h}$ is obtained for $\tilde{t}_{1}^{(2)}=\tilde{t}_{1}+\Delta \tilde{t}$, where $\Delta \tilde{t}=\Delta t / T_{p}$. Substituting Eq. (12) into the first double-summation term of Eq. (11) we obtain:

$$
\begin{aligned}
a f_{h}(\theta, \phi)=\frac{2 j}{h \pi} & \sin ^{2}\left(\frac{h \pi}{2}\right) e^{-j h \pi\left(2 \tilde{t}_{1}^{(1)}+1\right)} \cdot \operatorname{sgn}(h) \times \\
& \times\left(1+e^{-j 2 h \pi \Delta \tilde{t}} e^{j k_{0} D \sin \left(\theta+\theta_{\text {inc }}\right) \cos \left(\phi+\phi_{\text {inc }}\right)}\right) \times \\
& \times \sum_{n_{x}=1}^{N_{x}} e^{j k_{0}\left(n_{x}-1\right) p_{x} \sin \left(\theta+\theta_{\text {inc }}\right) \cos \left(\phi+\phi_{\text {inc }}\right)} \times \\
& \times \sum_{n_{y}=1}^{N_{y}} e^{j k_{0}\left(n_{y}-1\right) p_{y} \sin \left(\theta+\theta_{\text {inc }}\right) \sin \left(\phi+\phi_{\text {inc }}\right)} .
\end{aligned}
$$

As shown in Fig. 5(a), the entire DoA estimating metasurface consists of many DoA-MTS macrocells, say $M$, and, thus, the full array factor describing the scattering pattern of the entire metasurface is:

$$
A F_{h}(\theta, \varphi)=\sum_{m=1}^{M} a f_{h}(\theta, \phi) e^{j 2 k_{0}(m-1) D \sin \left(\theta+\theta_{i m c}\right) \cos \left(\phi+\phi_{\text {mic }}\right)}
$$

where $M$ is the number of DoA-MTS macro-cells.

We can notice now an interesting term in Eq. (13), and thus also in Eq. (14), that allows us enabling the spatial asymmetry in the scattered patterns for the two first-order harmonics of the reflected field: depending on the value of $h= \pm 1$, identifying the upper- and lower-sideband, the term $1+e^{-j 2 h \pi \Delta \tilde{t}} e^{j k_{0} D \sin \left(\theta+\theta_{\text {inc }}\right) \cos \left(\phi+\phi_{\text {inc }}\right)}$ exhibits two different values in the complex exponential, which unbalances the scattering pattern around the direction $\left[-\theta_{i n c} ;-\phi_{i n c}\right]$.

\section{Metasurface-BASEd DoA ESTIMATION METHOD}

\section{A. ID DoA estimation method based on DSB metasurface}

Let us start deriving the DoA estimation equations in the case of illuminating plane waves, whose wavevectors lie on the $x z$-plane, i.e., $\phi_{i n c}=0$. The goal is to estimate the performances of the proposed system in identifying the angle $\theta_{i n c}$ of the incident plane wave, i.e., $1 D$ DoA estimation.

In this scenario, the uniformity along the $y$-direction relaxes the need to consider the other scattering directions besides the ones laying on the $x z$-plane. Setting, thus, $\phi=0$, and observing the metasurface in broadside $(\theta=0)$, where the detecting antenna is located, the amplitude ratio between $A F_{-1}\left(\theta_{i n c}, \phi=0\right)$ and $A F_{+1}\left(\theta_{i n c}, \phi=0\right)$ can be written as:

$$
\begin{aligned}
\left|\frac{A F_{-1}}{A F_{+1}}\right| & =\left|\frac{a f_{-1}}{a f_{+1}}\right|=\left|\frac{1+e^{+j 2 \pi \Delta \tilde{t}} e^{j k_{0} D \sin \theta_{i c c}}}{1+e^{-j 2 \pi \Delta t} e^{j k_{0} D \sin \theta_{i n c}}}\right|= \\
& =\frac{\sqrt{1+\cos \left(k_{0} D \sin \theta_{i n c}+2 \pi \Delta \tilde{t}\right)}}{\sqrt{1+\cos \left(k_{0} D \sin \theta_{i n c}-2 \pi \Delta \tilde{t}\right)}}=\frac{\left|\cos \left(\frac{1}{2} k_{0} D \sin \theta_{i n c}+\pi \Delta \tilde{t}\right)\right|}{\left|\cos \left(\frac{1}{2} k_{0} D \sin \theta_{i n c}-\pi \Delta \tilde{t}\right)\right|} .
\end{aligned}
$$

Using trigonometric angle sum and difference identities to expend the right hand side of Eq. (15), and it can be simplified to obtain the DoA estimation curve as:

$$
g\left(f\left(\theta_{\text {inc }}\right)\right)=\tan \left(\frac{1}{2} k_{0} D \sin \theta_{\text {inc }}\right)=\frac{\left|A F_{1}\right|-\left|A F_{-1}\right|}{\left|A F_{1}\right|+\left|A F_{-1}\right|} \frac{\cos (\pi \Delta \tilde{t})}{\sin (\pi \Delta \tilde{t})},
$$

from which, we can estimate the DoA angle $\theta_{i n c}$ as: 


$$
\begin{aligned}
\theta_{i n c} & =g^{-1}\left(f^{-1}\left(\theta_{i n c}\right)\right)= \\
& =\operatorname{arcSin}\left(\frac{2}{k_{0} D} \operatorname{arcTan}\left(\frac{\left|A F_{1}\right|-\left|A F_{-1}\right|}{\left|A F_{1}\right|+\left|A F_{-1}\right|} \frac{\cos (\pi \Delta \tilde{t})}{\sin (\pi \Delta \tilde{t})}\right)\right) .
\end{aligned}
$$

To avoid angle ambiguity when Eq. (17) is used for DoA estimation, the curve should be monotonic among the estimation range, thus the conditions for no angle ambiguity are expressed as:

$$
-\frac{\pi}{2} \leq \frac{1}{2} k_{0} D \sin \theta_{i n c} \pm \pi \Delta \tilde{t} \leq \frac{\pi}{2}
$$

In the range of direction estimation $\theta_{i n c} \in[0 ; \pi / 2]$, the sine function $\sin \theta_{i n c}$ is real and within $[0 ;+1]$. Substituting $k_{0}=2 \pi / \lambda_{0}$ into (18), to avoid angle ambiguity, the conditions can be written as:

$$
|\Delta \tilde{t}| \leq \frac{1}{2}-\frac{D}{\lambda_{0}}
$$

Eq. (19) defines a bound for the time delay between the modulating signals to be applied for a given electrical dimension $D / \lambda_{0}$ of the Sub-macrocell. However, it is worth remembering that, due to the periodicity of the modulating signals $u_{2}(t)$, the time delay is always within a period, i.e., $|\Delta \tilde{t}| \leq 1 / 2$. This sets also an upper-bound for the sub-microcell dimension $D$, that cannot exceed the half-a-wavelength width.

\section{B. 2D DoA estimation extension}

The above method can be also extended to 2D DoA estimation. The $2 \mathrm{D}$ detection scheme requires that the scattering pattern splitting of the $1^{\text {st }}$-order harmonics of the reflected field is introduced for both $x$ - and $y$-directions of the metasurface to estimate both polar coordinates $\left[\theta_{i n c} ; \phi_{i n c}\right]$. This can be obtained by modulating the metasurfaces separately along the two main axes, $x$ - and $y$-directions. For example, the 1D modulation scheme reported in Sec. II-C can be applied in sequence along the $x$ - and $y$-directions by properly controlling in real-time the modulation profile on the metasurface or, alternatively, it is possible to use two identical DoA metasurfaces arranged orthogonally, as shown in Fig. 6.

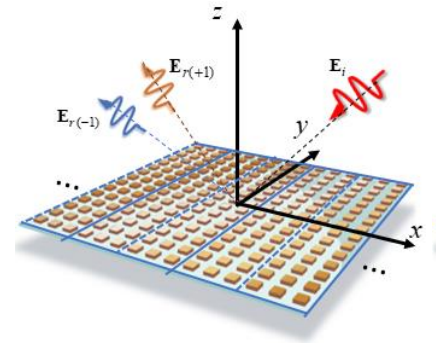

(a)

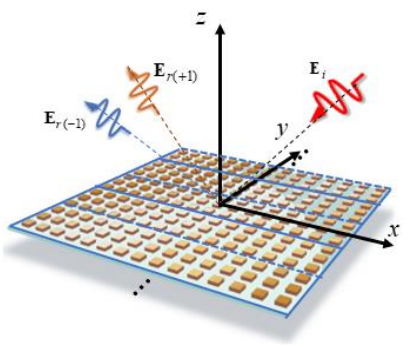

(b)
Figure 6. The 2D DoA estimation arrays (a) the first array along $\mathrm{x}$-direction, (b) the second array along $\mathrm{y}$-direction.

Following the same procedure illustrated in Sec. III-A for the 1D DoA estimation, we can derive the estimating function for the spatial modulation along the $x$-direction (Fig. 6(a)) considering an incident plane wave impinging from the direction $\left[\theta_{i n c} ; \phi_{i n c}\right]$. Being the detection direction $\theta=\varphi=0$, where the detecting antenna is located, we can write:

$$
\begin{aligned}
g_{x}\left(f_{x}\left(\theta_{i n c}, \varphi_{i n c}\right)\right) & =\tan \left(\frac{1}{2} k_{0} D \sin \theta_{i n c} \cos \varphi_{i n c}\right) \\
& =\frac{\left|A F_{1 x}\right|-\left|A F_{-1 x}\right|}{\left|A F_{1 x}\right|+\left|A F_{-1 x}\right|} \frac{\cos (\pi \Delta \tilde{t})}{\sin (\pi \Delta \tilde{t})}
\end{aligned}
$$

that returns:

$$
\sin \theta_{i n c} \cos \varphi_{i n c}=\frac{2}{k_{0} D} \arctan \left(\frac{\left|A F_{1 x}\right|-\left|A F_{-1 x}\right|}{\left|A F_{1 x}\right|+\left|A F_{-1 x}\right|} \frac{\cos (\pi \Delta \tilde{t})}{\sin (\pi \Delta \tilde{t})}\right)
$$

Moreover, the estimating function for the spatial modulation along the y-direction (Fig. 6(b)) is:

$$
\begin{aligned}
g_{y}\left(f_{y}\left(\theta_{i n c}, \varphi_{i n c}\right)\right) & =\tan \left(\frac{k_{0} D \sin \theta_{i n c} \sin \varphi_{i n c}}{2}\right)= \\
& =\frac{\left|A F_{1 y}\right|-\left|A F_{-1 y}\right|}{\left|A F_{1 y}\right|+\left|A F_{-1 y}\right|} \frac{\cos (\pi \Delta \tilde{t})}{\sin (\pi \Delta \tilde{t})}
\end{aligned}
$$

that, in turn, returns:

$$
\sin \theta_{i n c} \sin \varphi_{i n c}=\frac{2}{k_{0} D} \arctan \left(\frac{\left|A F_{1 y}\right|-\left|A F_{-1 y}\right|}{\left|A F_{1 y}\right|+\left|A F_{-1 y}\right|} \frac{\cos (\pi \Delta \tilde{t})}{\sin (\pi \Delta \tilde{t})}\right)
$$

From Eq. (21) and (23), $\theta_{i n c}$ and $\phi_{i n c}$ can be derived in closed forms as:

$$
\varphi_{\text {inc }}=\arctan \left[\frac{\arctan \left(\frac{\left|A F_{1 x}\right|-\left|A F_{-1 x}\right|}{\left|A F_{1 x}\right|+\left|A F_{-1 x}\right|} \frac{\cos (\pi \Delta \tilde{t})}{\sin (\pi \Delta \tilde{t})}\right)}{\arctan \left(\frac{\left|A F_{1 y}\right|-\left|A F_{-1 y}\right|}{\left|A F_{1 y}\right|+\left|A F_{-1 y}\right|} \frac{\cos (\pi \Delta \tilde{t})}{\sin (\pi \Delta \tilde{t})}\right)}\right],
$$

$$
\theta_{i n c}=\arcsin \left[\frac{2}{k_{0} D \cos \phi_{i n c}} \arctan \left(\frac{\left|A F_{1 x}\right|-\left|A F_{-1 x}\right|}{\left|A F_{1 x}\right|+\left|A F_{-1 x}\right|} \frac{\cos (\pi \Delta \tilde{t})}{\sin (\pi \Delta \tilde{t})}\right)\right]
$$

The 2D DoA estimation results can be expressed as Eq. (26), when $\theta$ is positive, $\theta=\theta, \phi=\phi$, but if $\theta$ is negative, $\theta=-\theta, \phi=$ $\phi+\pi$.

$$
(\theta, \phi)= \begin{cases}(\theta, \phi), & \theta>0 \\ (-\theta, \phi+\pi), & \theta<0\end{cases}
$$

\section{Accuracy of the DoA estimation method}

In this Section, the DoA estimation accuracy is evaluated using the estimation formulas of the incidence angles $\left[\theta_{i n c} ; \phi_{i n c}\right]$ derived in Sec. III-A and Sec. III-B. A Monte Carlo simulation is used to analyze the estimation accuracy, when the received signals have certain noise. This numerical simulation investigates the Mean Square Error (MSE) between the estimated and actual direction of incidence for the 1D DoA case. A plane wave at frequency $f_{0}$ illuminates the metasurface in the $x z$-plane $\left(\phi_{i n c}=0^{\circ}\right)$ with different incident angles, ranging from $\theta_{i n c}=-80^{\circ}$ to $\theta_{i n c}=+80^{\circ}$, with a step of $5^{\circ}$. The DoA space-time modulated metasurface consists of only one DoA-MTS macrocell, composed, in turn, by two sub-microcells with $D=0.44 \lambda_{0}$ and the time delay fraction $\Delta \tilde{t}=0.05$. The modulation frequency is $f_{m}=0.3 \times 10^{-3} f_{0}$. The signal-to-noise ratio (SNR) is set as $10 \mathrm{~dB}$ with respect to the 
amplitude of the total reflecting field from the metasurface. For each incident angle, a 1000-times Monte Carlo simulation is used to calculate the MSE of the estimation. The simulation results are shown in Fig. 7(a), where it can be found that the error is lower than $0.4^{\circ}$ over the entire field-of-view. Much lower error is found near $\pm 60^{\circ}$, whereas relative larger errors are found near $0^{\circ}, \pm 90^{\circ}$. The accuracy of the proposed method is linked to the slope of the DoA estimation curve (defined in Eq. 16) as shown in Fig.7(b). When the noise induces the same level error to $g\left(f\left(\theta_{i n c}\right)\right)$, the range of retrieved $\theta_{i n c}$ is dependent on the slope of the DoA estimation curve. That is to say if the slope is steeper, the retrieved range is smaller and DoA estimation is more accurate. On the contrary, if the slope is flatter, the retrieved range is wider and the accuracy becomes lower.

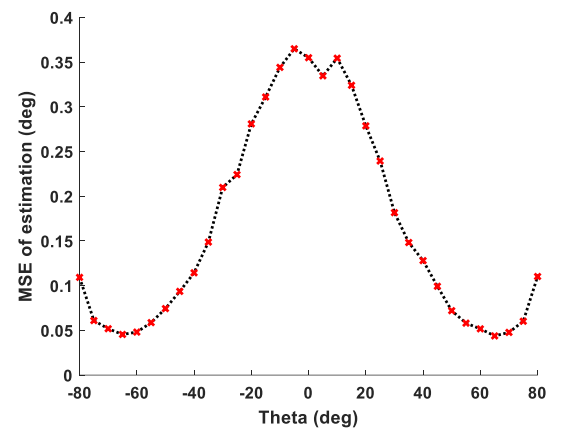

(a)

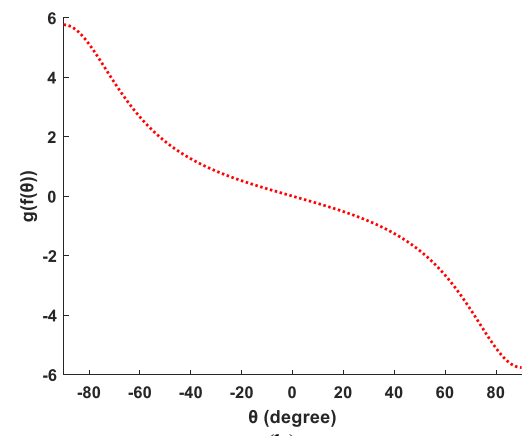

(b)

Figure 7. (a) Mean Square Errors in degrees of the DoA estimation under different illumination directions, (b) the corresponding analytical DOA estimation curve by two Sub-microcells with $D=0.44 \lambda_{0}$.

\section{DOA ESTIMATION USING A REALISTIC SPACE-TIME MODULATED METASURFACE}

To validate the proposed method based on a space-time modulated metasurface, a realistic metasurface structure is here considered and properly modulated for achieving the excitation of the $\pm 1^{\text {st }}$-order harmonics and the splitting of their scattering patterns. The metasurface should be able to exhibit unitary amplitude and a binary 0 - $\pi$ phase of the reflection coefficient over a field-of-view as wide as possible.

In Figs. 8(a)-(b), a single-polarized inclusion is proposed, which consists of adjacent patches placed on top of a Rogers $3010\left(\varepsilon_{r} \approx 10.2\right.$ ) dielectric substrate and connected through PIN diodes (BAR 65-03W) [58], which has two operational states, $\mathrm{ON}$ and $\mathrm{OFF}$, controlled by a bias voltage (3.3V or $0 \mathrm{~V})$..
The dimensions of the unit-cell hosting the inclusion are $p_{x}=10 \mathrm{~mm}$ and $p_{y}=10 \mathrm{~mm}$, and the height is $h_{s}=5.2 \mathrm{~mm}$, other parameters are $w 1=1.59 \mathrm{~mm}, l 1=3.5 \mathrm{~mm}, w 2=0.5 \mathrm{~mm}$ and $l 2=1 \mathrm{~mm}$.

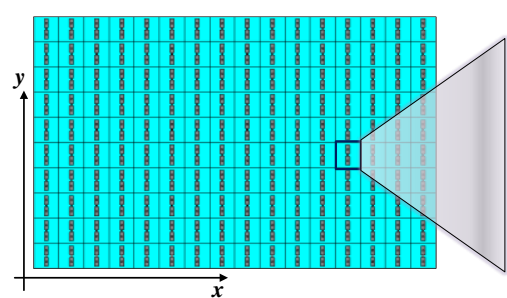

(a)

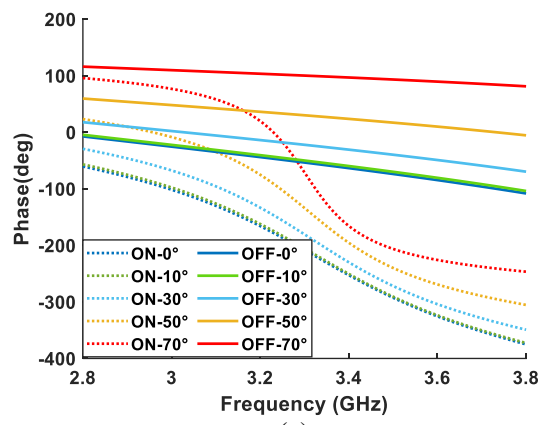

(c)

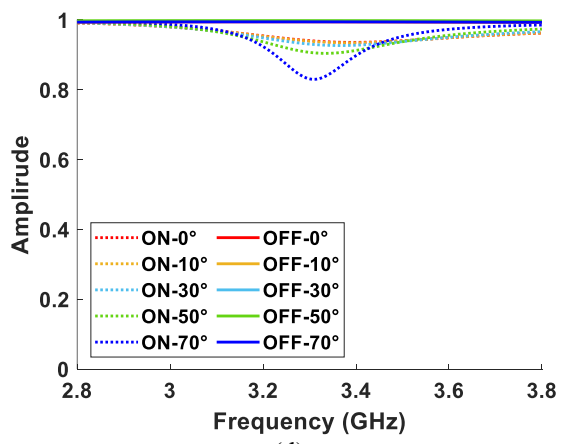

(d)

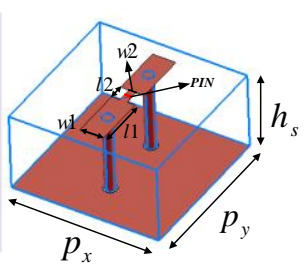

(b)
Figure 8. The binary metasurface and the reflection response of the unit-cell, (a) sketch of the metasurface composed of $10 \times 16$ unit-cells, (b) geometry of the 1-bit unit cell, (c) reflection phase and (d) reflection amplitude of the unit-cell as a function of frequency for the four states " 0 ", " $\pi$ ", under different illumination directions.

To show the 1-bit phase performance of the designed unit-cell under different operation states of the PIN diodes, we perform the full-wave simulation with CST Studio Suite [59]. in our simulation, when the PIN diode is at the ON state, it can be equivalent as a series circuit of parasitic inductance $(1.61 \mathrm{nH})$ and resistance $(0.7 \mathrm{Ohm})$; if the PIN diode is at the OFF state, a series circuit of parasitic inductance $(1.39 \mathrm{nH})$, capacitance $(0.41 \mathrm{pF})$, and resistance $(1.41 \mathrm{Ohm})$ is used to describe the physical model. In addition, a Floquet port is used to produce $y$-polarized waves incident onto the meta-atom and receive the reflected waves. Periodic boundary condition is set to its four sides to model the infinite array. It exhibits the binary 0 - $\pi$ phase response at $3.3 \mathrm{GHz}$, with a reflection amplitude larger than 0.82 over the angular range $\left[0^{\circ}-70^{\circ}\right]$ of the incident angles (Fig. 8 (c)-(d)). 
A. 1D DoA estimation using different widths of the DoA macrocell

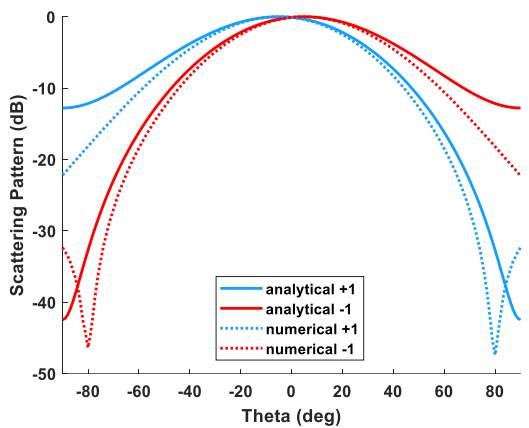

(a)

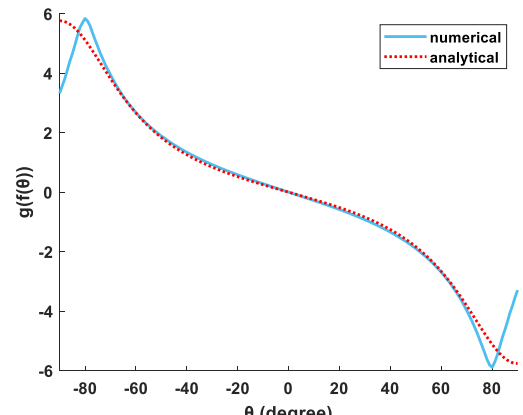

(b)

Figure 9. (a) Normalized scattering patterns of +1 and -1 order harmonics for the DOA estimation: comparison between analytical and numerical with the $\left[-90^{\circ}, 90^{\circ}\right]$ estimation range, (b) the corresponding DOA estimation curve, when array is $10 \times 8$ and $N_{x}=4$.

To demonstrate the DoA estimation using the proposed DSB space-time-modulated metasurface, a simulation is implemented considering a metasurface with one DoA-MTS microcell, composed by two sub-macrocells, each of which including $N_{x}=4$ and $N_{y}=10$ unit-cells shown in Fig. 8(b) along the $x$ - and $y$-directions, respectively. The operative central frequency is $f_{0}=3.3 \mathrm{GHz}$ and the modulation frequency is $f_{m}=1 \mathrm{MHz}$.

At the operative frequency, the periodicity of the 1-bit unit-cell along both axis is $p_{x, y}=0.11 \lambda_{0}$. According to the upper-bound limit defined in Eq. (19), the time delay $\Delta t$ between the modulating signals $u_{1}(t)$ and $u_{2}(t)$ must satisfy the relation $\Delta t \leq\left(0.5-N_{x} p_{x} / \lambda_{0}\right) T_{p}=0.06 T_{p}$. Here, the time delay has been thus set at $\Delta t=0.05 T_{p}$.

The normalized scattering patterns of $+1^{\text {st }}$ and $-1^{\text {st }}$-order harmonics are shown in Fig. 9(a), and the DoA estimation curve can be calculated from Eq. (16) by the scattering patterns of $+1^{\text {st }}$ - and $-1^{\text {st }}$-order harmonics. In Fig. 9(b), we show that the analytical and numerical curves agree very well except for the boundary of the incident angle range.

The DoA estimation results are summarized in Table I, where it is worth noticing that the proposed method returns very small absolute errors within the range $\left[-70^{\circ}, 70^{\circ}\right]$ between actual and estimated DoA. The other directions, out of this range, return a wrong estimation, due to the deterioration of the phase/amplitude responses of the unit-cell and the coupling effects among the unit-cells.

TABLE I

$N_{X}=4$, DOA ESTIMATION RESULTS

\begin{tabular}{ccc}
\hline \hline Direction $[\mathrm{deg}]$ & Estimation $[\mathrm{deg}]$ & Abs. Error $[\mathrm{deg}]$ \\
\hline 0 & 0.01 & 0.01 \\
+10 & 11.39 & 1.39 \\
+20 & 21.65 & 1.65 \\
+30 & 31.74 & 1.74 \\
+40 & 41.47 & 1.47 \\
+50 & 50.65 & 0.65 \\
+60 & 59.85 & 0.15 \\
+70 & 70.26 & 0.26 \\
-10 & -11.15 & 1.15 \\
-20 & -21.46 & 1.46 \\
-30 & 31.81 & 1.81 \\
-40 & 41.52 & 1.52 \\
-50 & -50.64 & 0.64 \\
-60 & -59.69 & 0.31 \\
-70 & -70.23 & 0.23 \\
\hline \hline
\end{tabular}

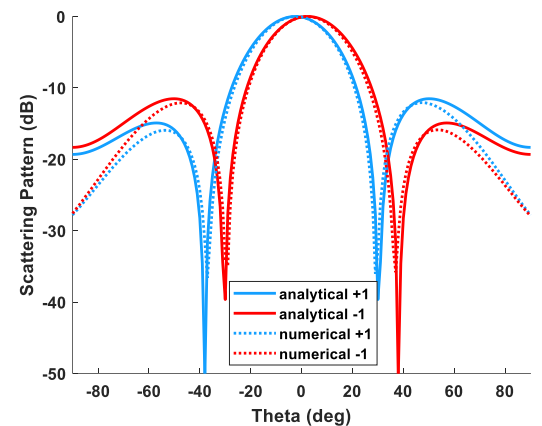

(a)

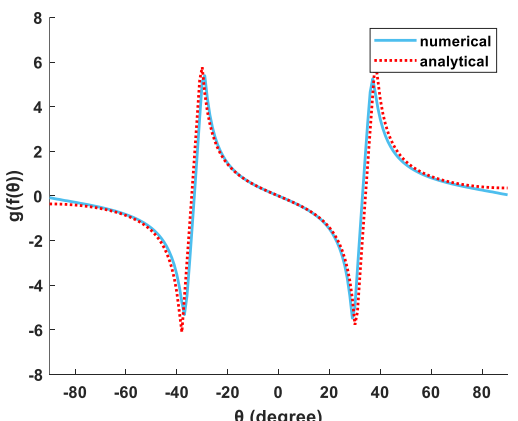

(b)

Figure 10. (a) Normalized scattering patterns of +1 and -1 order harmonics for the DOA estimation: comparison between analytical and numerical with the $\left[-30^{\circ}, 30^{\circ}\right]$ estimation range. (b) the corresponding DOA estimation curve when array is $10 \times 16$ and $N_{x}=8$.

It is interesting to also consider a narrower field-of-view and derive the corresponding modifications to the modulation scheme at the metasurface level. For example, limiting the field-of-view to the range $\left[-30^{\circ}, 30^{\circ}\right]$, using Eq. (19) we obtain 
that DoA estimation can be done using a larger width of the sub-macrocell. In particular, using again $\Delta t=0.05 T_{p}$, the width $D$ can be doubled, allowing to include $N_{x}=8$ unit cells along the $x$-direction and $N_{y}=10$ unit cells along the $y$-direction within a single sub-microcell. The metasurface, therefore, is composed by $10 \times 16$ unit-cells.

The normalized scattering patterns of $+1^{\text {st }}-$ and $-1^{\text {st }}$-order harmonics and the DoA estimation curve are reported in Fig. 10. In the angular range of interest, the curve is monotonic and single-valued, but it is steeper than the one in Fig. 9(b). This implies an enhancement of the estimation accuracy, as shown in Table II.

TABLE II

$N_{X}=8$, DOA ESTIMATION RESULTS

\begin{tabular}{ccc}
\hline \hline \multicolumn{3}{c}{$N X=8$, DoA Es. Error [deg] } \\
\hline Direction [deg] & Estimation [deg] & 0.05 \\
\hline 0 & 0.05 & 0.17 \\
+10 & 9.83 & 0.25 \\
+20 & 20.25 & 0.65 \\
-10 & 29.35 & 0.20 \\
-20 & -9.80 & 0.29 \\
-30 & -20.29 & 0.63 \\
\hline \hline
\end{tabular}

\section{B. ID DoA estimation using different time delays}

According to Eq. (16), the DoA estimation curve is not only related to the dimensions of the DoA element, but also to time delay $\Delta t$ of the modulating signals $u_{1}(t)$ and $u_{2}(t)$ applied to the two Sub-macrocells of the DoA-MTS microcell. To demonstrate the impact of the time delay in the DoA estimation accuracy, a simulation is implemented considering a metasurface composed by just one DoA-MTS microcell, whose sub-microcells include $N_{x}=4$ and $N_{y}=10$ unit-cells along the $x$ - and $y$-directions, respectively.

In Fig. 11, we report the DoA estimation curves for different time delays $\Delta t$. The light blue curve represents all the results for any value of $\Delta t \leq 0.05 T_{p}$ that satisfies Eq. (19). In this case, the curve is monotonic, and there is no ambiguity on the DoA. On the contrary for larger time delays (dashed curves in Fig. 11), the operational field of view shrinks according to Eq. (19), where the angle $\theta$ must be smaller for satisfying the constrain.

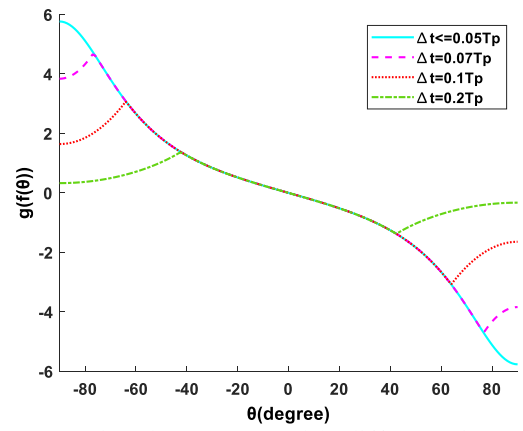

Figure. 11 DOA estimation curves with different time delay fractions, when array is $10 \times 8$ and $N_{x}=4$.

\section{CONCLUSION}

In this paper, we have proposed a DoA estimation method based on space-time modulated metasurfaces that exploits their capabilities to generate several harmonics in the scattered field, each of which is radiated towards different direction. By detecting the field in the broadside direction by using a single receiving antenna the metasurfaces-based DoA system can estimate in real-time the impinging angle of an illuminating monochromatic plane wave. The proposed approach exploits the amplitude unbalance of the received fields at broadside at only the two first-order harmonic frequencies generated by the interaction between the incident plane wave and the modulated metasurface. The analytical model for estimating the incident angle has been presented and successfully used in combination with a realistic 1-bit reflecting metasurface. The accuracy of 1D DoA estimation is verified through full-wave numerical simulations. Compared to conventional DoA estimation methods, the proposed one simplifies the computation and hardware complexity, ensuring a very good estimation accuracy. The proposed approach may have potential applications in wireless communications, target recognition and identification.

\section{REFERENCES}

[1] H. Krim, M. Viberg. "Two decades of array signal processing research: the parametric approach." IEEE Signal Process Mag., vol. 13, no. 4, pp. 67-94, 1996.

[2] H. L. Van Trees. "Detection, Estimation, and Modulation Theory, Part IV: Optimum Array Processing." New York, NY, USA: Wiley, 2002.

[3] H. Bayer, A. Krauss, T. Zaiczek, R. Stephan, O. Enge-Rosenblatt, and M. A. Hein, "Ka-band user terminal antennas for satellite communications [antenna applications corner]," IEEE Antennas Propag. Mag., vol. 58, no. 1, pp. 76-88, 2016.

[4] A. Kuchar, M. Tangemann, and E. Bonek, "A real-time doa-based smart antenna processor," IEEE Trans. Veh. Technol., vol. 51, no. 6, pp. 1279-1293, 2002.

[5] M. Burtowy, M. Rzymowski, and L. Kulas, "Low-profile espar antenna for rss-based doa estimation in iot applications," IEEE Access, vol. 7, pp. 17 403-17 411, 2019.

[6] M. Koivisto, M. Costa, J. Werner, K. Heiska, J. Talvitie, K. Lepp“anen, V. Koivunen, and M. Valkama, "Joint device positioning and clock synchronization in $5 \mathrm{~g}$ ultra-dense networks," IEEE Trans. Wireless Commun., vol. 16, no. 5, pp. 2866-2881, 2017.

[7] B. Liao and S. C. Chan, "Direction finding with partly calibrated uniform linear arrays," IEEE Trans. Antennas Propag., vol. 60, no. 2, pp. 922-929, Feb. 2012.

[8] R. Zhou, H. Zhang, and H. Xin, "Improved two-antenna direction finding inspired by human ears," IEEE Trans. Antennas Propag., vol. 59, no. 7, pp. 2691-2697, Jul. 2011.

[9] H. Cox, R. Zeskind, and M. Owen, "Robust adaptive beamforming," IEEE Trans. Acoust., Speech, Signal Process., vol. 35, no. 10, pp. 1365-1376, 1987.

[10] R. Roy and T. Kailath, "Esprit - Estimation of Signal Parameters Via Rotational Invariance Techniques," IEEE T Acoust Speech, vol. 37, no. 7, pp. 984-995, Jul. 1989.

[11] J. Steinwandt, F. Roemer, and M. Haardt, "Generalized least squares for esprit-type direction of arrival estimation," IEEE Signal Process. Lett., vol. 24, no. 11, pp. 1681-1685, 2017.

[12] R. O. Schmidt, "Multiple emitter location and signal parameterestimation," IEEE Trans. Antennas Propag., vol. 34, no. 3, pp. 276-280, Mar. 1986.

[13] D. Kundu, "Modified music algorithm for estimating DOA of signals," Signal processing, vol. 48, no. 1, pp. 85-90, 1996.

[14] P. Rocca, M. A. Hannan, M. Salucci and A. Massa, "Single-snapshot DoA estimation in array antennas with mutual coupling through a multiscaling BCS strategy," IEEE Trans. Antennas Propag., vol. 65, no. 6, pp. 3203-3213, June 2017. 
[15] Y. Ra'di, V. S. Asadchy, and S. A. Tretyakov, "Tailoring Reflections From Thin Composite Metamirrors,” IEEE Trans. Antennas Propag., vol. 62, no. 7, pp. 3749-3760, Jul. 2014.

[16] V. S. Asadchy, M. Albooyeh, S. N. Tcvetkova, A. Díaz-Rubio, Y. Ra’di, and S. A. Tretyakov, "Perfect control of reflection and refraction using spatially dispersive metasurfaces," Phys. Rev. B, vol. 94, no. 7, p. 075142, Aug. 2016.

[17] V. G. Ataloglou and G. V. Eleftheriades, "Arbitrary Wave Transformations with Huygens' Metasurfaces through Surface-Wave Optimization," pp. 1-5, 2021.

[18] A. Epstein and G. V. Eleftheriades, "Huygens' metasurfaces via the equivalence principle: design and applications," J. Opt. Soc. Am. B, vol. 33, no. 2, p. A31, Feb. 2016.

[19] D. Ramaccia, D. L. Sounas, A. V. Marini, A. Toscano, and F. Bilotti, "Electromagnetic isolation induced by time-varying metasurfaces: non-reciprocal bragg grating," IEEE Antennas and Wireless Propag. Lett., vol. 19, no. 11, pp. 1886-1890, May 2020.

[20] D. Ramaccia, D. L. Sounas, A. Alu, A. Toscano, and F. Bilotti, "Phase-Induced Frequency Conversion and Doppler Effect with Time-Modulated Metasurfaces," IEEE Trans. Antennas Propag., vol. 68, no. 3, pp. 1607-1617, Mar. 2020.

[21] Z. Wu and A. Grbic, "Serrodyne Frequency Translation Using Time-Modulated Metasurfaces," IEEE Trans. Antennas Propag., vol. 68, no. 3, pp. 1599-1606, Mar. 2020.

[22] M. Liu, D. A. Powell, Y. Zarate, and I. V. Shadrivov, "Huygens' Metadevices for Parametric Waves," Phys. Rev. X, vol. 8, no. 3, p. 031077, Sep. 2018.

[23] S. Glybovski, S. Tretyakov, P. Belov, Y. Kivshar, C. Simovski, "Metasurfaces: From microwaves to visible," Physics Reports, Vol. 634, pp. 1-72, 2016.

[24] D. Ramaccia, S. Arcieri, A. Toscano, and F. Bilotti, "Core-Shell Super-Spherical Nanoparticles for LSPR-Based Sensing Platforms," IEEE J. Sel. Top. Quantum Electron., vol. 23, no. 2, pp. 380-387, Mar. 2017.

[25] D. Ramaccia, A. Toscano, and F. Bilotti, "Scattering and absorption from super-spherical nanoparticles: analysis and design for transparent displays [Invited]," J. Opt. Soc. Am. B, vol. 34, no. 7, p. D62, Jul. 2017.

[26] S. Vellucci, A. Monti, M. Barbuto, A. Toscano and F. Bilotti, "Waveform-Selective Mantle Cloaks for Intelligent Antennas," IEEE Trans. Antennas and Propag., vol. 68, no. 3, pp. 1717-1725, March 2020.

[27] C. Huang, B. Sun,W. Pan, X. G. Luo, "Dynamical beam manipulation based on 2-bit digitally-controlled coding metasurface," Sci. Rep., vol. 7, no. 1, pp. 1-8, Feb. 2017.

[28] K. Chen, Y. Feng, F. Monticone, J. Zhao, B. Zhu, T. Jiang, and C. W. Qiu, "A reconfigurable active Huygens' metalens," Adv. Mater., vol. 29, no. 17, May 2017, Art. no. 1606422.

[29] W. Tang, J. Y. Dai, M. Chen, X. Li, Q. Cheng, S. Jin, K.-K. Wong, and T. J. Cui, "Programmable metasurface-based RF chain-free 8 PSK wireless transmitter," Electron. Lett., vol. 55, no. 7, pp. 417-420, Apr. 2019.

[30] M. Francesco and A. Alu. "Metamaterial, plasmonic and nanophotonic devices." Reports on Progress in Physics, vol. 80, no. 3, Art. no. 036401, 2017.

[31] L. Cong, P. Pitchappa, C. Lee, and R. Singh, "Active phase transition via loss engineering in a terahertz MEMS metamaterial," Adv. Mater., vol. 29, no. 26, Jul. 2017, Art. no. 1700733.

[32] Z. Miao, Q. Wu, X. Li, Q. He, K. Ding, Z. An, and L. Zhou, "Widely tunable terahertz phase modulation with gatecontrolled graphene metasurfaces," Phys. Rev. X, vol. 5, no. 4, 2015, Art. no. 041027.

[33] B. Y. Wei, W. Hu, Y. Ming, F. Xu, S. Rubin, J. G. Wang, and Y. Q. Lu, "Generating switchable and reconfigurable optical vortices via photo patterning of liquid crystals," Adv. ater., vol. 26, no. 10, pp. 1590-1595, Mar. 2014.

[34] L. Zhang, X. Q. Chen, S. Liu, Q. Zhang, J. Zhao, J. Y. Dai, and T. J. Cui, "Space-time-coding digital metasurfaces," Nat. Commun., vol. 9, no. 1, pp. 1-11, Oct. 2018.

[35] Z. Wu, C. Scarborough, and A. Grbic. "Space-Time-Modulated Metasurfaces with Spatial Discretization: Free-Space N-Path Systems." Phys. Rev. Appl., vol. 14, no. 6, Art. no. 064060, Dec. 2020.

[36] X. Fang, M. Li, D. Ding, F. Bilotti, and R. Chen, "Design of in-phase and quadrature two paths space-time-modulated metasurfaces," TechRxiv, 2021 [Online]. Available: 10.36227/techrxiv.15086538.v1.
[37] D. Ramaccia, A. Toscano, and F. Bilotti, "Light propagation through metamaterial temporal slabs: Reflection, refraction, and special cases," Opt. Lett., vol. 45, no. 20, 2020.

[38] D. Ramaccia, A. Alù, A. Toscano, and F. Bilotti, "Temporal multilayer structures for designing higher-order transfer functions using time-varying metamaterials," Appl. Phys. Lett., vol. 118, no. 10, p. 101901, Mar. 2021.

[39] V. Pacheco-Peña and N. Engheta, "Effective medium concept in temporal metamaterials," Nanophotonics, vol. 9, no. 2, pp. 379-391, Feb. 2020.

[40] V. Pacheco-Peña and N. Engheta, "Anti-reflection temporal coatings," Optica, vol. 7, no. 4, pp. 323-331, Mar. 2020.

[41] H. Li and A. Alù, "Temporal switching to extend the bandwidth of thin absorbers," Optica, vol. 8, no. 1, p. 24, Jan. 2021.

[42] D. Ramaccia, D. L. Sounas, A. Alù, A. Toscano, and F. Bilotti, "Doppler cloak restores invisibility to objects in relativistic motion," Phys. Rev. B, vol. 95, no. 7, p. 075113, Feb. 2017.

[43] J. W. Zang, D. Correas-Serrano, J. T. S. Do, X. Liu, A. Alvarez-Melcon, and J. S. Gomez-Diaz, "Nonreciprocal wavefront engineering with time-modulated gradient metasurfaces," Phys. Rev. Appl., vol. 11, no. 5, Art. no. 054054, May 2019.

[44] A. E. Cardin, S. R. Silva, S. R. Vardeny, W. J. Padilla, A. Saxena, A. J. Taylor, and A. K. Azad. "Surface-wave-assisted nonreciprocity in spatio-temporally modulated metasurfaces," Nat. Commun., vol. 11, no. 1, pp. 1-9, Nov. 2020.

[45] S, Taravati and G. V. Eleftheriades, "Full-duplex nonreciprocal beam steering by time-modulated phase-gradient metasurfaces," Phys. Rev. Appl. vol. 14, no. 1, Art. no. 014027, Jul. 2020.

[46] Y. Hadad, J. C. Soric, and A. Alu. "Breaking temporal symmetries for emission and absorption," Proceedings of the National Academy of Sciences, vol. 113, no. 13, pp. 3471-3475, Mar. 2016.

[47] L. Zhang, Z. X. Wang, R. W. Shao, J. L. Shen, X. Q. Chen, X. Wan, and T. J. Cui, "Dynamically realizing arbitrary multi-bit programmable phases using a 2-bit time-domain coding metasurface," IEEE Trans. Antennas and Propag., vol. 68, no. 4, pp. 2984-2292, Apr. 2020.

[48] J. Y. Dai, W. Tang, L. X. Yang, X. Li, M. Z. Chen, J. C. Ke, and T. J.Cui, "Realization of multi-modulation schemes for wireless communication by time-domain digital coding metasurface," IEEE Trans. Antennas and Propag., vol. 68, no. 3, pp. 1618-1627, Nov. 2019.

[49] J. A. Hodge, K. V. Mishra, and A. I. Zaghloul. "Intelligent time-varying metasurface transceiver for index modulation in 6G wireless networks," IEEE Antennas and Wireless Propag. Lett., vol. 19, no. 11, pp. 1891-1895, Sep. 2020.

[50] Q. Wu and R. Zhang. "Towards smart and reconfigurable environment: Intelligent reflecting surface aided wireless network," IEEE Commun. Mag., vol. 58, no. 1, pp. 106-112, Jan. 2019.

[51] X. Wang and C. Caloz, "Spread-spectrum selective camouflaging based on time-modulated metasurface," IEEE Trans. Antennas and Propag., vol. 69, no. 1, pp. 286-295, Jan. 2021.

[52] J. Manley and H. Rowe, "Some General Properties of Nonlinear Elements-Part I. General Energy Relations," Proc. IRE, vol. 44, no. 7, pp. 904-913, Jul. 1956.

[53] H. Rowe, "Some General Properties of Nonlinear Elements. II. Small Signal Theory,” Proc. IRE, vol. 46, no. 5, pp. 850-860, May 1958.

[54] C. He, X. Liang, Z. Li, J. Geng and R. Jin, "Direction Finding by Time-Modulated Array With Harmonic Characteristic Analysis," IEEE Antennas and Wireless Propag. Lett., vol. 14, pp. 642-645, 2015.

[55] Y. Youn, J. Kim, S. Oh, and S. -H. Yi, "Time-modulated array system controlled with bipolar squared periodic sequence for direction of arrival estimation," IEEE Wireless Commun. Lett., vol. 10, no. 9, pp. 1895-1898, Sept. 2021.

[56] A. M. Yao, W. Wu, and D. G. Fang. "Single-side band time-modulated phased array," IEEE Trans. Antennas and Propag., vol. 63, no. 5, pp. 1957-1968, Feb. 2015.

[57] C. Balanis, Antenna theory: analysis and design, $4^{\text {th }}$ edition, Wiley, 2006.

[58] Infineon BAR65-03W PIN Diode, datasheet and models available at: https://www.infineon.com/cms/en/product/rf-wireless-control/rf-diode/rf -pin-diode/antenna-switch/bar65-03w/.

[59] "CST - Computer Simulation Technology." [Online]. Available: https://www.cst.com/. [Accessed: 12-Apr-2019]. 